\title{
Philippe de Mézières, rhétorique et poétique, édité par J. BLANCHARD
}

\section{Paola Cifarelli}

\section{(2) OpenEdition}

\section{Journals}

\section{Édition électronique}

URL : https://journals.openedition.org/studifrancesi/31408

DOI : $10.4000 /$ studifrancesi.31408

ISSN : 2427-5856

\section{Éditeur}

Rosenberg \& Sellier

\section{Édition imprimée}

Date de publication : 1 août 2020

Pagination : 381-383

ISSN : 0039-2944

\section{Référence électronique}

Paola Cifarelli, «Philippe de Mézières, rhétorique et poétique, édité par J. BLANCHARD », Studi Francesi [En ligne], 191 (LXIV | II) | 2020, mis en ligne le 01 septembre 2020, consulté le 18 septembre 2021. URL : http://journals.openedition.org/studifrancesi/31408; DOI : https://doi.org/10.4000/studifrancesi. 31408

Ce document a été généré automatiquement le 18 septembre 2021.

\section{(c)}

Studi Francesi è distribuita con Licenza Creative Commons Attribuzione - Non commerciale - Non opere derivate 4.0 Internazionale. 


\title{
Philippe de Mézières, rhétorique et poétique, édité par J. BLANCHARD
}

\author{
Paola Cifarelli
}

\section{RÉFÉRENCE}

Philippe de Mézières, rhétorique et poétique, édité par J. BLANCHARD avec la collaboration de R. BLUMENFELD-KOSINSKI et A. CALVET, Genève, Droz, 2019, «Cahiers d'Humanisme et Renaissance» 157,325 pp.

1 Ce volume consacré à Philippe de Mézières réunit treize contributions réparties en cinq sections, qui constituent une réflexion sur la poétique du discours méziérien dans ses formes différentes; en effet, si la plupart des études concernent le Songe du vieil pelerin, tout le corpus des œuvres du Vieux Pèlerin est analysé ici dans cette perspective. Le but, explicité dans l'Introduction par Joël Blanchard, est de rechercher les tendances stylistiques, les procédés rhétoriques, les registres, le répertoire d'images qui constituent «la marque propre du Solitaire» (p.11) par-delà la langue utilisée ou le genre textuel pratiqué.

Dans la première section («La rhétorique dans tous ses états»), les proverbes et les formules à allure proverbiale du Songe du vieil pelerin font l'objet d'une analyse qui prend en compte les structures servant à leur insertion dans le texte, les expressions qui y sont associées, l'instance à laquelle ils sont parfois attribués, la créativité dont ils témoignent et la fonction qu'ils exercent dans le cadre du projet réformateur de Philippe de Mézières (Maria colombo timeld, 'Qui tient le moien, il va le seur chemin' ("Songe du vieil pelerin", 1133, 28-29). Les 'proverbes' dans le "Songe” de Philippe de Mézières, pp.31-50); deux Annexes offrent un relevé complet respectivement des séquences qualifiées de 'proverbe' par Mézières et des formules qui se rapprochent à un proverbe médiéval attesté.

3 Michelle szKILNIK, quant à elle, analyse les traces d'une prose rythmique dans le texte du Songe; elle poursuit et complète les études antérieures du prologue, ayant mené à 
formuler l'hypothèse de l'existence d'un texte en vers perdu (Rimes, Ryhtmes et couleurs de rhétorique dans "Le songe du vieil pelerin", pp. 51-69); des passages particulièrement significatifs pour témoigner de l'existence de rimes dans la prose sont extraits du chap. 5 du livre I et du chap. 51 du livre II, tandis que d'autres passages du livre I montrent l'existence de fragments versifiés soulignant d'autres effets de rythme, bouleversant l'ordre syntaxique ou attestant de «couples sonores» (p.60) situés à des endroits stratégiques de la phrase ou concentrés dans le discours de personnages particuliers. L'A. interprète ces procédés comme des tentatives de transposer dans la langue vernaculaire les techniques de la prose poétique latine, plutôt que comme le résultat d'un dérimage.

4 La section II («Stratégies oratoires») réunit trois articles, dont le premier (Sébastien CAZALAS, "Je suis Triboullee jusques au ventre et au cuer». L'écriture de la lamentation dans quelques œuvres de Philippe de Mézières et de Jean Juvénal des Ursins, pp. 73-90) analyse les stratégies d'utilisation de la plainte chez deux auteurs qui, par-delà les différences qui les séparent, sont proches pour leur rôle auprès des puissants, leurs idées politiques et leur foi sincère: le corpus analysé, comprenant l'Épître au roi Richard II (1395) et l'Épitre lamentable et consolatoire (1396-1397) de Philippe de Mézières, montre que l'écriture de la douleur nécessite une grande maîtrise rhétorique et une utilisation savante de l'écriture métaphorique, sans pourtant renoncer à la sincérité de l'émotion.

5 La contribution de Jean-Claude müHLETHALER (Par-delà la tristesse et l'indignation: modulations affectives et portée de la voix satirique chez Philippe de Mézières, pp. 91-118) a comme objet le langage des émotions, et particulièrement de l'indignation, vu comme déclencheur de la satire; une comparaison entre l'Arbre des batailles d'Honoré Bovet et trois ouvrages de Philippe de Mézières (Songe, Chevalerie de la Passion du Christ, Epistre lamentable et consolatoire) met en évidence que la satire est à la fois un instrument du processus de légitimation d'une réforme de la Chrétienté et un moyen pour réaliser ce projet ambitieux à travers la dynamique de l'argumentation.

6 Le rôle de Philippe de Mézières dans le processus de reconfiguration du champ littéraire caractérisant le XIV siècle, et particulièrement ses expérimentations pour la définition d'«auctorialité», sont au cœur de l'étude d'Estelle DOUDET (Philippe de Mézières, orateur: les nouveaux territoires d'une posture d'auteur, pp.119-133); les modalités d'utilisation dans des contextes différents du terme «orateur» pour désigner son statut d'auteur, et la signification lourde de sens que Philippe de Mézières attribue à ce terme font apparaitre une progression dans la posture d'écrivain qu'il adopte de plus en plus consciemment, avec des nuances inédites et «promises à un riche avenir» (p. 133).

7 La troisième section, consacrée à «L'herméneutique méziérienne», s'ouvre sur la contribution de Philippe FRIEDEN (Exercices de lecture: l'usage de l'allégorie dans le "Songe du Viel Pelerin", pp.137-161), qui analyse le procédé de l'allégorie d'un point de vue exégétique; le caractère problématique de l'écriture allégorique dans le Songe est abordé à l'aide du concept d'allégorèse et mis en parallèle avec le Livre du chemin de longue estude de Christine de Pizan d'une part, et le Quadriloge invectif d'Alain Chartier d'autre part. La structure agonistique de ces deux textes et leur stratégie visant à fournir une synthèse entre des arguments différents sert de clé de lecture pour le Songe, dans lequel le processus de la lecture est conçu comme exercice d'interprétation et de décryptage.

8 Ce sont encore le Songe du vieil pelerin et le procédé rhétorique de l'allégorie qui font l'objet de l'article de Daisy DELOGU (Allegory, semiotics and salvation: the parable of the 
talents in the "Songe du veil pelerin", pp. 165-185); l'interprétation que saint Grégoire a fournie de la parabole des talents constituerait la base du programme allégorique de Philippe de Mézières, fondé sur une «hermeneutically- and spiritually-fruitful analogy between semiotics, economics, and sacramental structures or networks» (p. 164). En particulier, l'utilisation du terme «bezant», et plus généralement les mots appartenant au réseau sémantique de la monnaie, sont conçus comme une structure herméneutique susceptible de permettre à Philippe de connecter la dimension spirituelle, politique, éthique dans son ouvrage.

9 La mise en page de la Table dans l'Oratio tragedica et surtout de celle du manuscrit Paris, Ars. 2682-2683 du Songe, destinées à guider le lecteur et le préparer à l'exégèse du texte, est analysée de manière méticuleuse par Andrea TARNOWSKI (Philippe de Mézières, all at once (allegory and the visual), pp.187-203); l'A., qui prend en compte également les illustrations du codex, montre à quel point Philippe de Mézières était attentif aux aspects visuels de balisage du texte pour rendre plus compréhensible le message profond de cet ouvrage.

10 La quatrième section («Théâtralité et rites») contient deux articles; le premier (Helen SWIFT, "La devise et forme singuliere de la fin du Povre Pelerin»: ritual configuration and rhetorical invention in Philippe de Mézière's “Testament” (1392), pp. 207-225) est consacré au texte que Philippe de Mézières composa à l'adresse des Célestins pour leur exposer ses volontés quant au traitement de son propre corps et de son âme à l'heure du trépas. La singularité de ce discours sur la mort tant par rapport à d'autres textes contemporains sur l'art de bien mourir, tel le De arte moriendi de Gerson, qu'en relation au reste de la production méziérienne, se révèle n'être qu'apparente, surtout à l'égard du Songe et de sa fiction allégorique.

11 Dans la deuxième contribution de cette section, Adrian ARMSTRONG (Griselda, the musical? From "Livre de la vertu du sacrement de mariage" to "L'Estoire de Griseldis en rimes et par personnages", pp. 227-247) se penche sur l'Estoire de Griseldis en rime et par personnages, adaptation dramatique de la traduction de la nouvelle boccacienne de Griselda que Philippe de Mézières avait insérée dans son Livre de la vertu de sacrement de mariage. Loin de considérer ce texte comme médiocre, l'A. montre sa qualité à travers une analyse des techniques d'utilisation du vers, et particulièrement de la rime mnémothechnique (qu'il propose de désigner «shared couples»); ce procédé de versification se révèle porteur d'une charge dramaturgique très forte, tout comme les cinq passages en «triplet rhyme» (p. 335) et les enjambements. Pareillement, la création d'un petit public interne à l'action dramatique et agissant sur scène, ainsi que l'accentuation de la valeur illocutoire et perlocutoire de certains dialogues ou les insertions musicales, ont une fonction performative essentielle que l'approche «éclectique» adoptée ici met en évidence de manière claire.

La section conclusive («Poétique de l'alchimie chez Philippe de Mézières») réunit trois études. Catherine GAULLIER-BOUGASSAS (L'Écriture du miroir du prince au XIV siècle: "Le Songe du Vieil Pèlerin" de Philippe de Mézières et le "Secretum secretorum", pp. 251-269) aborde le Songe du point de vue de son appartenance au genre du miroir du prince, en suggérant que le regard porté par Philippe de Mézières sur les sciences occultes, objet d'une véritable stigmatisation, peut être interprété comme une réaction au discours d'Aristote dans le Secretum Secretorum, best-seller de la littérature médiévale que Philippe n'a pas pu ignorer. Tout en cherchant de substituer, dans le modèle de l'union $\mathrm{du}$ roi et du philosophe, l'image de ce dernier par celle du prophète, Philippe remplace 
les sciences occultes par une «sainte alchimie», spirituelle et capable de réformer la chrétienté. Le discours alchimique associé à l'enseignement politique, vu comme un détournement des buts du miroir du prince, serait donc un aspect de la «réfutation implicite d'un des miroirs des princes à succès de son époque» (p. 269).

Isabelle FABRE (Le «Viel Soltaire» en son miroir: la poétique du "Livre de la vertu du sacrement de mariage" de Philippe de Mézières (c. 1385-1389) à la lumière du "Roman de la Rose", pp. 271-289) propose d'analyser le Livre de la vertu du sacrement de mariage comme un «miroir de l'âme» construit à partir du Roman de la Rose, conçu comme un modèle poétique et esthétique d'architecture déconcertante. L'A. analyse la métaphore du «vergier», allégorie du jardin spirituel dont les éléments clé sont la fontaine et le jardin des simples, puis elle étudie successivement la fonction du rubis et du diamant, symbole marial, et enfin l'allégorie de la navigation au compas, avec le parcours médical dessiné à travers la métaphore du bourdon. Dans son Livre Mézières transforme donc le sémantisme des motifs tirés du Roman de la Rose en fonction de son propre projet poétique et moral.

Le volume s'achève sur la contribution de Joël BLANCHARD et Antoine CALVET (L'«apothicairerie» de Philippe de Mézières, creuset d'une poétique nouvelle?, pp. 291-307) qui retrace le parcours existentiel de Mézières pour se concentrer sur l'Oratio tragedica, étape conclusive de cet itinéraire à la fois humain et spirituel. Ce texte est d'abord situé dans le contexte intellectuel où il fut conçu et réalisé, marqué par la Rhetorica divina de Guillaume d'Auvergne et par son modèle oratoire, le De inventione de Cicéron. Mézières y est décrit comme un digne héritier de l'orateur latin, bien que l'ouvrage soit classé comme une prière. La structure et le contenu de l'œuvre sont ensuite analysés et mis en parallèle avec d'autres textes contemporains, pour souligner les particularités et l'originalité du travail de Mézières tant au niveau de la matière, que de l'exégèse et de la poétique. 